\title{
Valor del índice de excentricidad del ventrículo izquierdo con tomografía spect de perfusión miocárdica en diversas condiciones.
}

\author{
Teresa Massardo ${ }^{1}$, Isabel Berrocal ${ }^{1}$, Gabriel Castro ${ }^{1}$, M. Paz Muñoz ${ }^{2}$, Natalia Santis ${ }^{2}$,
Pamela Padilla ${ }^{1}$, Hernán Prat ${ }^{3}$, Verónica Araya ${ }^{4}$. \\ 1. Sección Medicina Nuclear, Departamento Medicina, Hospital Clínico Universidad de Chile. \\ 2. Alumnas de Tecnología Médica, Facultad de Medicina, Universidad de Chile \\ 3. Departamento Cardiovascular del Hospital Clínico Universidad de Chile. \\ 4. Sección Endocrinología Departamento Medicina, Hospital Clínico Universidad de Chile. \\ Financiado parcialmente por Proyecto IAEA-CRP E13031
}

\section{Resumen:}

Introducción: El índice de excentricidad (IE) obtenido con SPECT miocárdico permite evaluación de esfericidad ventricular izquierda en remodelación patológica.

Objetivo: conocer IE en adultos con diversas condiciones cardíacas.

Método: Grupo I: Controles $(\mathrm{n}=44)$ con $>2$ factores de riesgo cardiovascular exceptuando Diabetes Mellitus (DM); Grupo II: DM 2 ( $\mathrm{n}=41)$. Ambos grupos asintomáticos sin enfermedad coronaria conocida. Grupo III: Con alteración de motilidad segmentaria $y / o$ infarto de miocardio $(\mathrm{n}=64)$. Grupo IV: Dilatados $(n=21)$ con alteraciones de motilidad difusa o miocardiopatías. Se utilizó Sestamibi-Tc99m y procesamiento QGS. (IE esfera valor $\approx 0$ ).

Resultados: Las fracciones de eyección del ven- trículo izquierdo fueron significativamente mayores en grupos I y II que en grupos III y IV y los volúmenes de fin de diástole menores. Los IE en reposo fueron: Grupo I: $0.889 \pm 0.003$; Grupo II: $0.873 \pm 0.03$; Grupo III: $0.846 \pm 0.04$ y Grupo IV: $0.820 \pm 0.06$. Prueba t de student entre Grupos: I vs II ( $\mathrm{p}=0.028)$; I vs III así como I vs IV ( $\mathrm{p}<0.0001)$. Sin embargo, el análisis multivariado ANOVA no mostró diferencia entre los grupos I vs II ni entre grupos III vs IV, aunque sí entre I y II vs III y IV. El IE fue menor en grupos con alteraciones de motilidad que sin estas $(\mathrm{p}<0.001)$. No hubo Controles del Grupo I con función normal con IE $<0.82$. Los hallazgos de IE post estrés fueron similares.

Conclusión: La cuantificación de IE mediante SPECT de perfusión miocárdica permite diferenciar grupos de pacientes con y sin alteraciones morfológicas, que pudiera utilizarse en evaluación de remodelación patológica.

Correspondencia:

Dra. Teresa Massardo V.

Sección Medicina Nuclear, Departamento de Medicina;

Hospital Clínico Universidad de Chile.

Santos Dumont 999-1E, Independencia,

Código Postal 6531063, Santiago-Chile

fono/fax: 56-2-7770569

tmassardo@redclinicauchile.cl 


\section{Value of left ventricular excentricity index obtained by myocardial perfusion tomography}

Background: The Eccentricity Index (EI) obtained with myocardial SPECT allows adequate assessment of left ventricular shape in pathological myocardial remodeling.

Aim: To compare EIs in adults with different cardiac conditions.

Methods: 170 patients were studied. Group I $(n=44)$ were control subjects with $>2$ cardiovascular risk factors not including Diabetes Mellitus (DM); Group II $(\mathrm{n}=41)$ had type II DM. Patients in both groups were asymptomatic without known coronary artery disease or wall motion abnormalities. Group III $(n=64)$ had segmental wall motion abnormality and/or myocardial infarction; Group IV ( $\mathrm{n}=21)$ included patients with dilated ventricles and diffuse abnormal wall motion. We used gated $99 \mathrm{mTc}$ Sestamibi SPECT and QGS processing (EI sphere $\approx 0$ ).

\section{Introducción}

La remodelación patológica del ventrículo izquierdo es un fenómeno explorado con gran interés en la enfermedad coronaria (EC) debido a su implicancia pronóstica y compleja terapia. Se presenta posterior a infarto de miocardio (IM) como fenómeno compensatorio; también en miocardiopatías, valvulopatías, diversas patologías congénitas e hipertensión pulmonar. El tipo de terapia también tiene efecto sobre el grado de remodelación y de la función ventricular ${ }^{1,2}$. Pacientes con hipertensión arterial pueden presentar remodelación significativa; la disfunción diastólica es un fenómeno asociado y corresponder a una alteración más precoz ${ }^{3}$.

Específicamente, en el IM la pérdida aguda de tejido miocárdico, de otras células y de la matriz extracelular ocasiona un abrupto incremento en las condiciones de estrés que induce una forma particular de remodelación en la zona infartada misma y en el miocardio distante, que además contribuye a la generación de disfunción ventricular y dilatación progresiva del ventrículo. Los mecanismos involucrados en la remodelación patológica son complejos e incluyen diversos factores de activación neurohormonales, de inflamación, de necrosis, así como de estrés oxidativo con alteraciones a nivel de miocitos, de fibroblastos, de otras células, así como del colágeno y de otros componentes de la matriz extracelular. Existen alteraciones fenotípicas involucradas; por ejemplo, se ha descrito recientemente fibrosis miocárdica como manifes-
Results: Left ventricular ejection fraction was significantly higher and end diastolic volumes lower in Groups I and II than in Groups III and IV. Resting EI was $0.889 \pm 0.003$ in Group I, $0.873 \pm 0.03$ in Group II, $0.846 \pm 0.04$ in Group III and $0.820 \pm 0.06$ in Group IV. Groups I and II had significantly higher EI, compared to Groups III and IV. The EI was lower in groups with abnormal wall motion compared to those without it $(\mathrm{p}<0.001)$. No patient in Group I with a normal LV function had an EI $<0.82$. EI obtained post stressed behaved in a way similar to resting EI in all groups.

Conclusion: Myocardial perfusion SPECT EI differs in patients with and without left ventricular morphological changes and could be helpful in the evaluation of left ventricular remodeling.

Key words: Left ventricular shape, Eccentricity index, miocardial SPECT.

tación temprana de miocardiopatía hipertrófica. El estado profibrótico en pacientes con mutaciones de sarcómero mostraron biomarcadores de colágeno más elevados que en controles asociado a alteraciones en resonancia magnética (RM) cardíaca ${ }^{4}$.

La revascularización coronaria mejora los fenómenos de remodelación, lo que se asocia a recuperación de función ventricular, evaluada con parámetros sistólicos: fracción de eyección ventricular izquierda (FEVI) y volúmenes ${ }^{5}$. Es importante mencionar que posterior a un IM la apertura de la arteria culpable disminuye la remodelación patológica ${ }^{6}$. La angioplastía primaria post IM disminuye la alteración de la contractilidad regional, pero no altera la deformación del ventrículo izquierdo en sístole ${ }^{7}$. Por otra parte, la remodelación reversa con entrenamiento mediante ejercicio es mayor en insuficiencia cardíaca no isquémica que en la de origen isquémico ${ }^{8}$.

Para diagnosticar alteraciones que hagan sospechar la remodelación miocárdica se debe evaluar la geometría cardiaca la que puede cuantificarse con varios índices y técnicas; la más común es la Ecocardiografía que calcula índice de excentricidad y circularidad ${ }^{9,10}$, basándose en un plano e incluyendo promedio de engrosamiento muscular en la fórmula ${ }^{2}$. La medición con ventriculografía contrastada es un método descrito principalmente en patología valvular aórtica y en IM que incluye también engrosamiento, lo cual traduce indirectamente un parámetro de estrés miocárdico ${ }^{11}$. La RM cardíaca evalúa índice de expansión y de esfericidad, así como radio de adelgaza- 
miento ${ }^{12}$. En el SPECT de perfusión miocárdica se han implementado índices de forma y de excentricidad (IE) 13,14; éste último utiliza las 3 dimensiones del ventrículo izquierdo. El IE se puede medir en imágenes isotópicas gatilladas y no gatilladas o sumadas, pero se prefiere utilizar el sístole para el cálculo. Este índice en diástole parece ser menos discriminativo ${ }^{2}$. El significado y forma de cálculo varía dependiendo de la técnica.

En cuanto a la terapia para atenuar los efectos del proceso fisiopatológico de remodelación ventricular, con el fin de mejorar el pronóstico de los pacientes con EC avanzada e IM, se han utilizado los inhibidores de la enzima convertidora de angiotensina, inhibidores de la aldosterona y tratamiento de la hipertensión crónica o la enfermedad valvular cardíaca de manera precoz ${ }^{15-17}$. Las nuevas técnicas de imagen molecular para evaluación de angiogénesis, apoptosis y alteraciones intersticiales durante remodelación cardíaca, pueden tener implicancia para el pronóstico y terapia de pacientes con insuficiencia cardíaca ${ }^{18}$.

El objetivo de este trabajo preliminar fue conocer si existe diferente IE en pacientes con diversas condiciones miocárdicas en adultos con y sin EC conocida; pero con factores de riesgo cardiovascular (FRCV).

\section{Material y Método}

\section{Población}

\section{Se estudió retrospectivamente a los siguientes grupos de} pacientes:

- Grupo I: (n=44) adultos mayores de 45 años considerados controles con más de 2 FRCV, exceptuando Diabetes Mellitus (DM). Fueron FRCV: la hipertensión arterial, presente en $77 \%$ de los casos, la dislipidemia, el tabaquismo y los antecedentes familiares de primer grado con EC.

- Grupo II: Diabéticos 2 (n=41). Mayores de 45 años y con al menos 5 años de DM conocida; con al menos 1 FRCV adicional, 68\% de ellos con hipertensión arterial.

Ambos grupos I y II no tenían EC conocida, eran asintomáticos coronarios y sin alteraciones de motilidad (en cualquier examen previo incluyendo el SPECT gatillado) y también los con electrocardiograma (ECG) de reposo sugerentes de EC. Se excluyeron asimismo pacientes con bloqueo de rama izquierda. Estos pacientes pertenecen a protocolo prospectivo en curso de pesquisa de isquemia en diabéticos asintomáticos. Se midió en forma automática en programa QPS $®$ el score diferencial sumado (SDS) en grupos I y II que correspondió a $2.4 \pm 2.8$ y 2.6 3 3.4 respectivamente con $\mathrm{p}=\mathrm{ns}$. Se considera significativo $\mathrm{SDS}>3$. No hubo defectos de perfusión de tipo fijo, informados como necrosis en estos grupos.

- Grupo III: Pacientes con EC que presentaran alteración de motilidad segmentaria y/o IM ( $n=64)$.

- Grupo IV: Pacientes Dilatados con alteración de motilidad difusa y/o miocardiopatía isquémica $(n=21)$.

Los grupos III y IV fueron reclutados del trabajo clínico habitual del centro de los que tenían coronario y ventriculografía contrastada previa o concomitante. Los casos se evaluaron considerando los antecedentes clínicos y las alteraciones de motilidad ventricular y volúmenes existentes en estudio SPECT de perfusión en reposo así como en el ECG de la prueba de estrés cardíaco.

En cada grupo, los hombres correspondieron a $64 \%$, $43 \%, 80 \%$ y $76 \%$ respectivamente; sin diferencia significativa de edad, con promedios de $57 \pm 10,60 \pm 7,63 \pm 12$, $63 \pm 13$ años, respectivamente; sólo los el grupo Control vs los IM fueron significativamente diferentes.

Criterios técnicos de inclusión: Señal de gatillado ECG estable durante la adquisición; estudios sin movimiento durante ésta o con cierto movimiento susceptible de adecuada corrección con software específico; estudios sin actividad significativa del radiofármaco en estructuras extra cardíacas, que dificultaran francamente la delimitación ventricular.

Protocolo de estrés: Los grupos Control y DM efectuaron estrés con protocolo de Bruce obteniendo al menos $85 \%$ de frecuencia cardíaca máxima; la mayoría de los pacientes de los otros grupos efectuó estrés farmacológico con dipiridamol i.v. en dosis alta de $0.84 \mathrm{mg} / \mathrm{kg}$, infundido en $4 \mathrm{~min}$.

Técnica Tomográfica SPECT: Se utilizó SestamibiTc99m y protocolo habitual de uno o dos días en equipo Siemens dual head, con arco de $180^{\circ}$ y dos colimadores de alta resolución y baja energía en $90^{\circ}$; gatillado en ambas fases de estrés y reposo. El software correspondió a versión preliminar [Siemens Medical. "Cedars Cardiac Suite: Quantitative evaluation of myocardial perfusion SPECT" and Siemens Medical Solutions. "e.soft-Cedars: Quantitative Evaluation of Myocardial Perfusion SPECT". 2003] con procesamiento en su modalidad automática y manual o semiautomático para estudio gatillado de función QGS (Quantitative Gated SPECT) en todos los casos y sólo automática para perfusión QPS (Quantitative Perfusion SPECT).

Índice de excentricidad (IE): Este índice también llamado de esfericidad es un parámetro morfológico que medido mediante el SPECT miocárdico permite cuantificación reproducible, especialmente si se efectúa automáticamente ${ }^{14}$. Se calcula en imagen sumada no gatillada y en sístole 
en el estudio gatillado; se efectúan diámetros en la mitad de la cavidad, en 3 planos con la fórmula mostrada en la Figura 1. El cálculo realizado en imágenes con delimitación manual de los bordes miocárdicos puede ser más ajustado a la realidad pero menos reproducible.

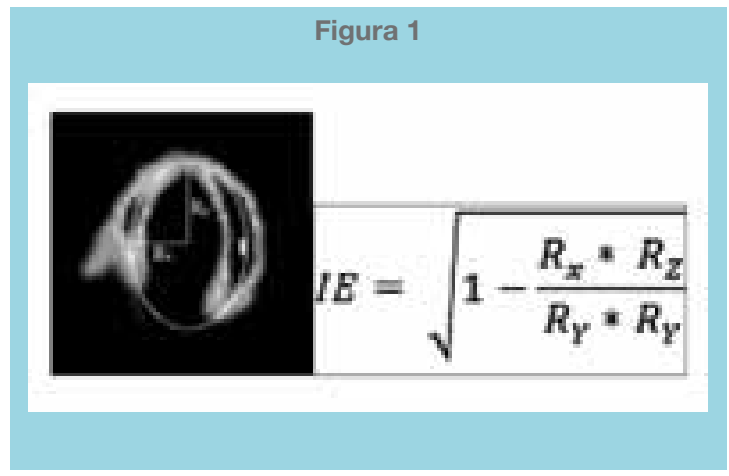

Fórmula de IE isotópico y ejes considerados para este efecto (mod. con autorización de Germano $G$ ) siendo $x, y$, z los diámetros tridimensionales). $I E=$ Esfera valor $=0$ y Línea valor $=1$

Análisis Estadístico: Se compararon los 4 grupos de pacientes y además se dividió paralelamente el grupo total de pacientes según un parámetro funcional del ventrículo izquierdo; aquellos con FEVI mayor o igual como a $45 \%$ y menor a $45 \%$ y con volumen de fin diástole (VFD) menor o igual a $120 \mathrm{ml}$ y mayor a $120 \mathrm{ml}$, valores utilizados como de corte de normal para el método y software empleado. Los promedios y desviaciones estándar de los datos fueron analizados con prueba $\mathrm{t}$ de student para muestras no pareadas con 2 colas (significancia p: 0.05) y analizados correlacionados con coeficiente de Pearson. Además, se efectuó análisis multivariado ANOVA que sería más apropiado por el tipo de muestra pequeño y no pareado.

\section{Resultados}

La Tabla 1 muestra los valores de función sistólica ventriculares izquierdos, con fracción de eyección (FEVI) y volúmenes de fin de diástole (VFD) y de sístole (VFS) así como los IE de reposo y post-estrés en los 4 grupos analizados con técnica gatillado manual. Las FEVI fueron significativamente diferentes entre los grupos con $\mathrm{p}<0.0001$, salvo grupo I vs II ( $\mathrm{p}=0.013)$ grupo III vs IV ( $\mathrm{p}=0.028)$; los volúmenes también fueron muy diferentes $(\mathrm{p} \leq 0.0002)$ salvo grupos I vs II y III vs IV que fueron similares ( $p=n s$ ). La distribución de los IE en reposo con sus respectivas significancias pueden observarse en la Figura 2 y los IE post-estrés en la Figura 3.
Tabla 1: Resumen de valores de función sistólica ventricular izquierda, con fracción de eyección (FEVI), volúmenes de $\mathrm{f} \mathbf{n}$ de diástole (VFD) y de sístole (VFS) así como los IE de reposo y post-estrés de gatillado manual en los 4 grupos analizados.

\begin{tabular}{|c|c|c|c|c|}
\hline & $\begin{array}{c}\text { I Controles } \\
(n=44)\end{array}$ & $\begin{array}{l}\text { II DM } \\
(n=41)\end{array}$ & $\begin{array}{c}\text { III IM } \\
(n=64) .\end{array}$ & $\begin{array}{c}\text { IV Dilatado } \\
(\mathrm{n}=21)\end{array}$ \\
\hline FEVI $(\%)^{*}$ & $57 \pm 10$ & $50 \pm 13$ & $36 \pm 11$ & $29 \pm 11$ \\
\hline VFD (ml) $)^{\#}$ & $78 \pm 19$ & $78 \pm 50$ & $127 \pm 70$ & $164 \pm 93$ \\
\hline VFS (ml) $\%$ & $35 \pm 15$ & $44 \pm 44$ & $86 \pm 6$ & $122 \pm 85$ \\
\hline IE reposo ${ }^{\&}$ & $0.889 \pm 0.0031$ & $0.873 \pm 0.03$ & $0.846 \pm 0.04$ & $0.820 \pm 0.06$ \\
\hline IE post-estrés ${ }^{\&}$ & $0.884 \pm 0.003$ & $0.865 \pm 0.04$ & $0.841 \pm 0.04$ & $0.818 \pm 0.05$ \\
\hline
\end{tabular}

*FEVI reposo: $p<0.02$, entre todos \#VFD reposo: $p=n s$ grupos $I \mathrm{vs}$.

II y III vs. IV

$\%$ VFS $p=n s ;$ sólo Grupo I vs II $\& p=n s$ en todos los grupos $(>0.05)$

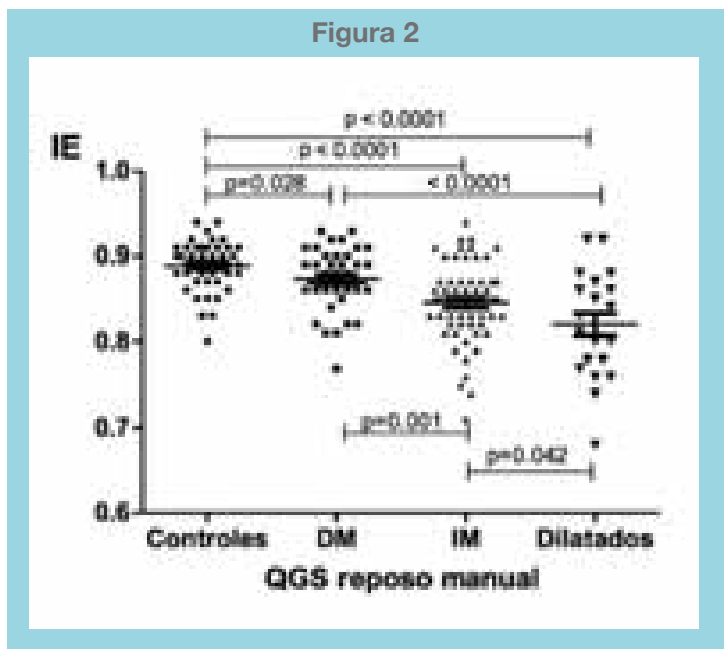

Distribución y significancia de los IE en reposo con método semiautomático o manual en los diversos grupos.

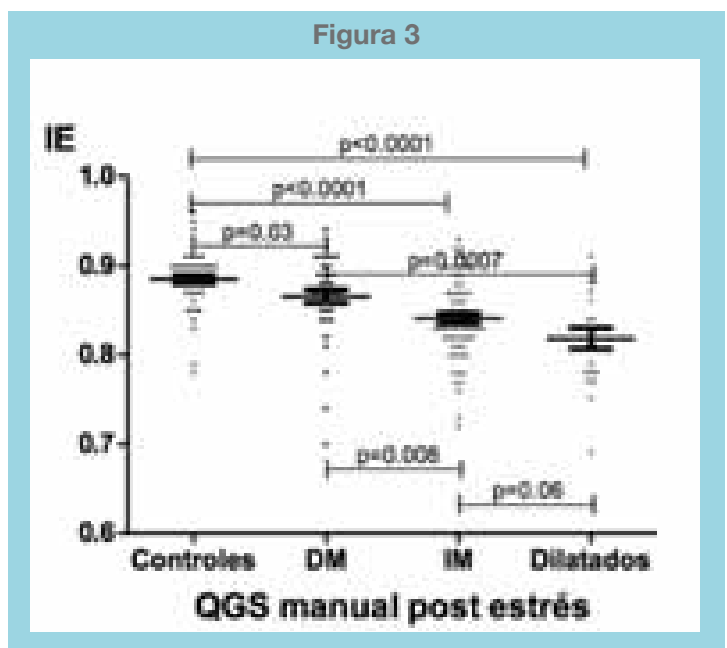

Distribución y significancia de los IE en post-estrés con método semiautomático o manual en los diversos grupos. 
El análisis de promedios mostró diferencia en la esfericidad medida con IE en reposo entre los grupos I y II ( $\mathrm{p}=0.028$ ); entre grupos I y III así como entre grupos I y IV ( $\mathrm{p}<0.0001$, en ambos). También hubo diferencias con IE post-estrés: entre grupo I y II $(\mathrm{p}=0.03)$ y entre grupos I y III y entre grupos I y IV $(\mathrm{p}<0.0001 \mathrm{en}$ ambos). Sin embargo, el análisis multivariado ANOVA no mostró diferencia en IE entre los grupos I y II ni entre grupos III y IV; sí hubo diferencia con este análisis en IE entre grupos Control y DM vs. Grupos IM y Dilata$\operatorname{dos}(\mathrm{p}<0.01 \mathrm{y}<0.001$, respectivamente).

Por otra parte, se encontró que, en reposo, no hubo casos del grupo Control con función normal que tuvieran IE $<0.82$ lo que se traduce en que pacientes con valores menores presentan alteración de forma global. En el procesamiento totalmente automático el valor correspondiente es IE $<0.74$. Al evaluar las diferencias entre el promedio de IE en reposo correspondiente a 0.812 \pm 0.055 de pacientes con alteraciones funcionales significativas considerando VFD $>120 \mathrm{ml}$ y $\mathrm{EF}<45 \%$ vs el promedio de IE $(0.888 \pm 0.026)$ de pacientes con volumen y función de VI normales para la técnica en uso, la p fue $<0.0001$.

Además, se encontró que en todos los grupos el IE fue similar post estrés que en reposo. Ver Tabla 1 $(\mathrm{p}>0.05)$; también hubo diferencia entre grupos con y sin alteración de motilidad $(\mathrm{p}<0.001)$, tanto en QGS manual como en QPS automático. Ver Figuras 4 y 5. El QGS automático no tuvo valor discriminativo por sobreposición de algunos controles aunque las diferencias del IE entre el grupo Control y los con alteraciones de motilidad segmentaria y difusa fueron muy significativas ( $\mathrm{p}=0.0004 \mathrm{y}<0.0001$, respectivamente).

Los IE obtenidos con procesamiento semiautomático o manual en reposo tuvieron rango entre 0.94 y 0.68 y los IE en post-estrés entre 0.96-0.69. Se observó la existencia de buena correlación entre estos $(r=0.84)$; el procesamiento automático de los mismos parámetros presentó cierta mayor dispersión tanto en estudios gatillados $(\mathrm{r}=0.71)$ como no gatillados $(\mathrm{r}=0.73)$; aunque todas las correlaciones fueron significativas $(\mathrm{p}<0.0001)$.

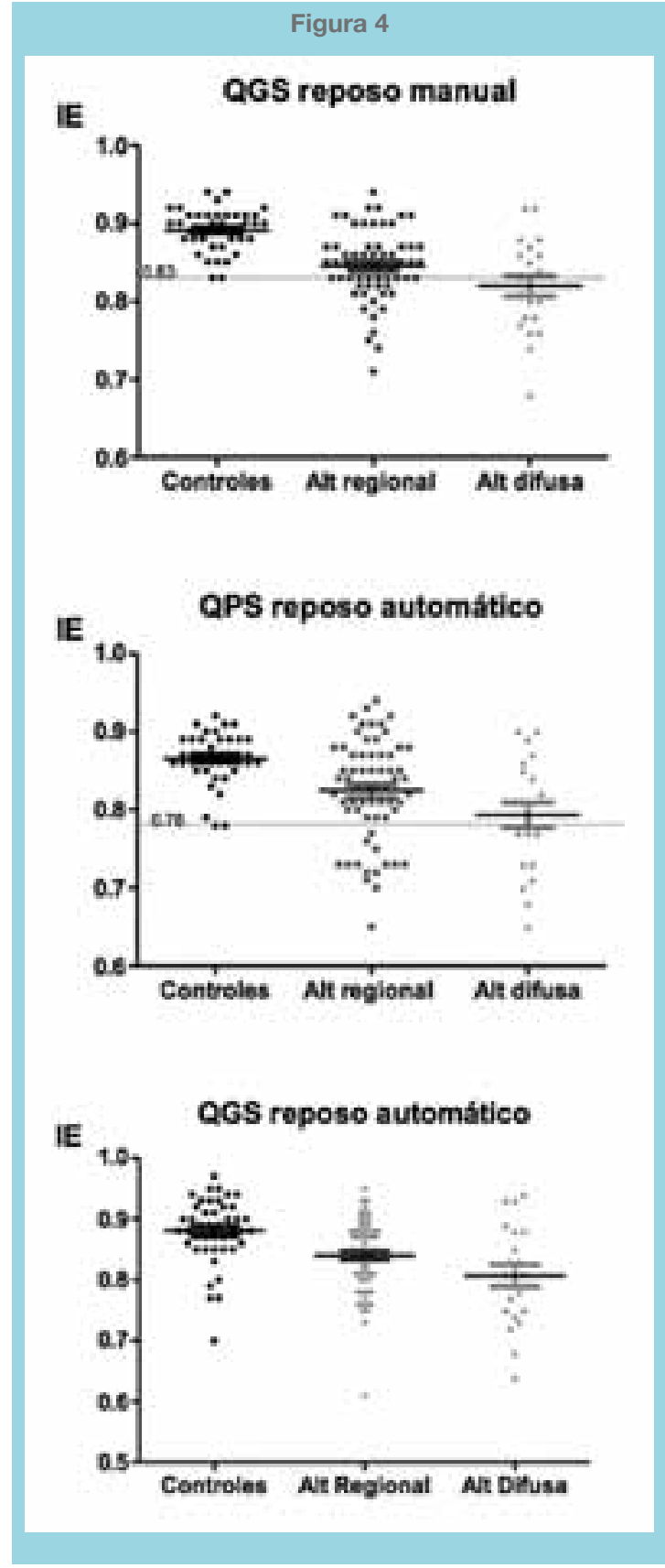

Distribución del IE en reposo según alteración de motilidad.

Ver valor de corte respecto a controles.

Superior: Procesamiento QGS manual, el valor de corte es altamente discriminativo: 0.83

Centro: Procesamiento QPS reposo automático, el valor discriminativo es algo menor 0.78 .

Inferior: Procesamiento QGS reposo automático, no hay valor discriminativo. 


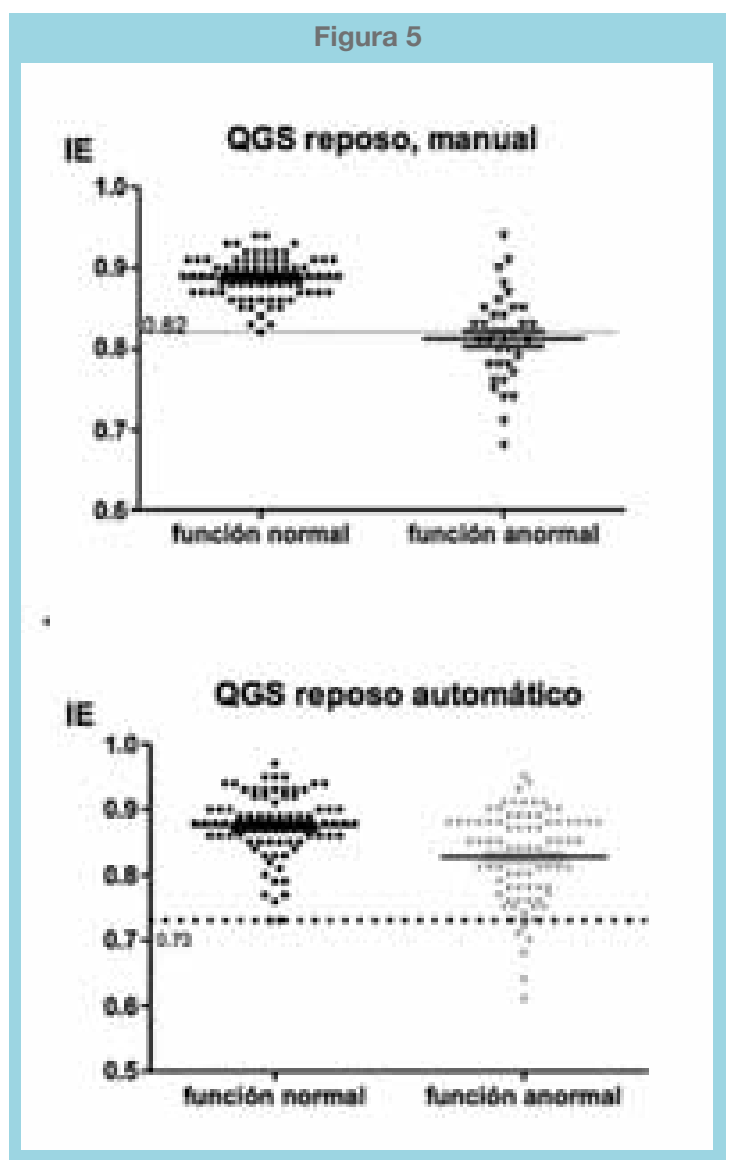

Distribución del IE reposo manual en grupos con y sin disfunción ventricular.

Superior: Procesamiento QGS manual. El valor de corte es altamente discriminativo y corresponde a 0.82 .

Inferior: Procesamiento QGS automático, el valor es menos discriminativo y corresponde a 0.73 .

\section{Discusión}

El contar con un índice simple y reproducible para medir excentricidad entre las herramientas disponibles en los exámenes SPECT de perfusión habituales ${ }^{19}$ parece muy útil. En nuestro trabajo comprobamos que el IE provisto por el software es adecuado siendo capaz de diferenciar pacientes con mayor esfericidad por su patología de base: los casos con mayor volumen y dilatación presentaron menor IE.

Nos parece interesante el hallazgo que los pacientes diabéticos con al menos 5 años de enfermedad tuvieran diferente IE que los casos del grupo Control no diabéticos con otros FRCV al efectuar la comparación simple, aunque con significancia sólo con prueba estadística simple. Los pacientes del grupo Control tenían FRCV, siendo en su gran mayoría hipertensos arteriales, patología que asociada a la hipertrofia secundaria también presenta remodelación ventricular y disfunción diastólica precoz, teniendo los DM mayor alteración en este sentido. Este hecho apunta a que diabéticos asintomáticos coronarios podrían tener ya cierto grado de remodelación en fase inicial. Los mecanismos fisiopatológicos de la hipertrofia muscular cardíaca en diversas condiciones es un fenómeno complejo que puede estar involucrado ${ }^{3,20-25}$. El encontrar diferencias en el IE al comparar pacientes con parámetros de función miocárdica normal, respecto a los con parámetros patológicos sustenta que la técnica tiene valor discriminativo (el IE fue mayor en aquellos con función normal, y en sentido contrario, es decir los pacientes con disfunción ventricular tuvieron menor IE, que se acerca más a cero, lo que implica mayor esfericidad).

A pesar de que la reproducibilidad del procesamiento automático debiera ser $100 \%$ en un mismo estudio, hay mayor dispersión al comparar diversos procesamientos del mismo paciente. Está claro que las variables asociadas especialmente a la excreción biliar del radiotrazador pueden requerir cierta intervención del operador; lo cual debe considerarse individualmente para seguimiento.

Debido a que no hemos encontrado en la revisión de la literatura valores normales de IE, una ventaja de este trabajo es describir un valor cercano a la normalidad pues aunque nuestros casos Control no son verdaderos normales ni tienen baja probabilidad de EC, por presentar más de un FRCV. Se debiera estudiar ese grupo en un trabajo posterior. Creemos que es más adecuado utilizar el IE medido en estudio gatillado con técnica manual sólo si es necesario para asegurar un buen ajuste de las paredes ventriculares que no siempre se obtiene con el método completamente automático. Este problema se encontró en este trabajo en menos del $20 \%$ de los casos, lo cual se confirma con el buen ajuste observado para los dos tipos de procesamiento QGS. En la imagen diastólica hay menos efecto de volumen parcial (problema técnico en pacientes con corazones pequeños, especialmente en mujeres normales); y al tener mayor tamaño la cavidad ventricular, pudiera ser más fácil para el software delimitar bordes.

Limitaciones: Una fue contar con una muestra pequeña y en especial de pacientes con dilatación. Otra, es el no haber incluido a verdaderos normales como controles podría haber llevado a una subestimación de las diferencias entre los valores controles y patológicos del IE; aunque no es simple, clínicamente, obtener ese tipo de sujetos a los cuales se les sometería a una dosis de irradiación baja aunque cuestionable. 


\section{Conclusiones:}

La cuantificación de IE mediante SPECT de perfusión miocárdica gatillada permite diferenciar diversos grupos de pacientes con y sin alteraciones morfológicas, lo que pudiera ser un aporte en la pesquisa o evaluación terapéutica de EC y/o miocardiopatías. La DM pareciera tener cierto grado de mayor esfericidad no significativa respecto a controles similares sin DM pero con FR cardiovasculares principalmente hipertensión arterial, lo que debiera confirmarse con mayor casuística.

Consideramos que la forma del ventrículo más esférica por remodelación lo que cambia el valor de la variable
IE entre grupos I y II (sin EC) vs II y IV (con EC) en forma no directamente dependiente de la función. Para la obtención del IE, debiera usarse el mismo método de procesamiento idealmente semiautomático estandarizado o manual y debido a que no existen diferencias entre reposo y en post-estrés (Tabla 1) bastaría usar el reposo. También recomendamos considerar el IE en el informe de los SPECT miocárdicos puesto que proporciona información predictiva relativa de la pérdida de capacidad funcional del ventrículo izquierdo en pacientes que padecen de EC.

Los autores no tienen conflicto de intereses con empresas comerciales ni de otro tipo.

\section{Referencias:}

1. CIRILLO M, AMADUCCI A, BRUNELLI F, DALLA TOMBA M, PARRELLAC P, TASCA G, et al. Determinants of postinfarction remodeling affect outcome and left ventricular geometry after surgical treatment of ischemic cardiomyopathy. J Thor Cardiov Surg. 2004; 127: 1648-1656.

2. VAN GELDORP IE, VANAGT WY, BAUERSFELD EU, TOMASKE M, PRINZEN FW, DELHAAS T. Chronic Left Ventricular Pacing Preserves Left Ventricular Function in Children. Pediatr Cardiol. 2009; 30:125-132.

3. RUIZ-HURTADO G, DELGADO C. Nitric oxide pathway in hypertrophied heart: new therapeutic uses of nitric oxide donors. J Hypertens. 2010; 28: S56-61.

4. HO CY, LÓPEZ B, COELHO-FILHO OR, LAKDAWALA NK, CIRINO AL, JAROLIM P, et al. Myocardial fibrosis as an early manifestation of hypertrophic cardiomyopathy. N Engl J Med. 2010; 363: 552-563.

5. SLART RH, BAX JJ, VAN VELDHUISEN DJ, VAN DER WALL EE, IRWAN R, SLUITER WJ, et al. Prediction of functional recovery after revascularization in patients with chronic ischaemic left ventricular dysfunction: head-to-head comparison between $99 \mathrm{mTc}$-sestamibi/18F-FDG DISA SPECT and 13N-ammonia/ 18F-FDG PET. Eur J Nucl Med Mol Imaging. 2006; 33: 716-723.

6. MARROQUIN OC, LAMAS GA. Beneficial effects of an open artery on left ventricular remodeling after myocardial infarction. Prog Cardiovasc Dis. 2000; 42: 471-483.

7. BARLETTA G, ANTONIUCCI D, BUONAMICI P, TOSO A, DEL BENE R, FANTINI F. Left ventricular shape and function in primary coronary angioplasty. Int $\mathrm{J}$ Cardiol.
2008; 125: 364-375.

8. DELAGARDELLE C, FEIEREISEN P, VAILLANT M, GILSON G, LASAR Y, BEISSEL J, et al. Reverse remodeling through exercise training is more pronounced in non-ischemic heart failure. Clin Res Cardiol. 2008; 97: 865-871.

9. RYAN T, PETROVIC O, DILLON JC, FEIGENBAUM H, CONLEY MJ, ARMSTRONG WF. An echocardiographic index for separation of right ventricular volume and pressure overload. J Am Coll Cardiol. 1985; 5: 918-27.

10. PORTMAN MA, BHAT AM, COHEN MH, JACOBSTEIN MD. Left ventricular systolic circular index: an echocardiographic measure of transseptal pressure ratio. Am Heart J. 1987; 114: 1178-1182.

11. FIENO DS, HILLENBRAND HB, REHWALD WG, HARRIS KR, DECKER RS, PARKER MA, et al. Infarct resorption, compensatory hypertrophy, and differing patterns of ventricular remodeling following myocardial infarctions of varying size. J Am Coll Cardiol. 2004; 43: 2124-2131.

12. DI DONATO M, BARLETTA G, MORI F, FANTINI F. Regional left ventricular wall motion abnormalities in chronic volume overload. Cathet Cardiovasc Diagn. 1983; 9: 453-462.

13. ABIDOV A, SLOMKAPJ, NISHINAH, HAYES SW, KANG $\mathrm{X}$, YODA $\mathrm{S}$, et al. Left ventricular shape index assessed by gated stress myocardial perfusion SPECT: Initial description of a new variable. J Nucl Cardiol 2006; 13: 652-659.

14. GERMANO G. Quantitative analysis in Myocardial SPECT imaging. In Quantitative Analysis in Nuclear Medicine Imaging, 2006, 15; 471-493

15. LANDMESSER U, WOLLERT KC, DREXLER H. Potential novel pharmacological therapies for myocardial remodeling. Cardiovasc Res. 2009; 81: 519-527. 
16. CHAREONTHAITAWEE P, CHRISTIAN TF, HIROSE K, GIBBONS RJ, RUMBERGER JA. Relation of initial infarct size to extent of left ventricular remodeling in the year after acute myocardial infarction. J Am Coll Cardiol.1995; 25: 567 573.

17. SUTTON MS, KEANE MG. Reverse remodeling in heart failure with cardiac resynchronisation therapy. Heart. 2007; 93: $167-171$

18. CANDELL-RIERA J, ROMERO-FARINA G, AGUADÉBRUIX S, CASTELL-CONESA J. Ischemic cardiomyopathy: a clinical nuclear cardiology perspective. Rev Esp Cardiol. 2009; 62:903-917.

19. GERMANO G, KAVANAGH PB, SLOMKAPJ, VAN KRIEKINGE SD, POLLARD G, BERMAN DS. Quantitation in gated perfusion SPECT imaging: The Cedars- Sinai approach. J Nucl Cardiol. 2007;14: 433-454.

20. FANTINI F, BARLETTA G, DI DONATO M, TOSO A, BARONI M. Alterations in left ventricular shape in patients with angina and single-vessel coronary disease. Coron Artery Dis.
1994; 5: 901-908

21. MIHL C, DASSEN WR, H. KUIPERS. Cardiac remodeling: concentric versus eccentric hypertrophy in strength and endurance athletes. Neth Heart J. 2008;16:129-133

22. TISCHLER M, ASHIKAGA T, LEWINTER M. Relation between left ventricular shape and Doppler filling parameters in patients with left ventricular dysfunction secondary to coronary artery disease Am J Cardiol.1995; 76: 553-556

23. HILL JA, OLSON EN. Cardiac Plasticity. N Engl J Med. 2008; 358: 1370-1380.

24. MAYTIN M, COLUCCI WS. Molecular and cellular mechanisms of myocardial Remodeling. J Nucl Cardiol. 2002; 9: 319-27.

25. KARAAHMET T, TIGEN K, DUNDAR C, PALA S, GULER A, KILICGEDIK A, et al. The effect of cardiac fibrosis on left ventricular remodeling, diastolic function, and $\mathrm{N}$-terminal pro-B-type natriuretic peptide levels in patients with nonischemic dilated cardiomyopathy. Echocardiography. 2010; 27: 954-960 